\title{
Fourier Domain Mode Locking Laser for Enhanced Sweeping Range Based on Dispersion-Shifted Fiber
}

\author{
Jianbing Xu, Rui Zhu, Xie Wang, Chi Zhang, P. C. Chui, and Kenneth K. Y. Wong * \\ Photonic Systems Research Laboratory, Department of Electrical and Electronic Engineering, \\ The University of Hong Kong, Pokfulam Road, Hong Kong. \\ *E-mail: kywong@eee.hku.hk
}

\begin{abstract}
A Fourier domain mode locking laser with dispersion management at longwavelength band is investigated. Sweeping bandwidth and obtained OCT axial resolution compared with the case without dispersion control is enhanced by $33 \%$ and $30 \%$, respectively.

(C) 2012 Optical Society of America

OCIS codes: (110.4500) Optical coherence tomography; (140.4050) Mode-locked lasers; (060.2400) Fiber properties.
\end{abstract}

\section{Introduction}

Recently, Fourier domain mode locking (FDML) swept laser [1] has been playing an increasingly important role in optical coherence tomography (OCT) [2]. OCT employing this kind of swept laser, also called swept source OCT (SS-OCT), can provide higher acquisition speed and improved sensitivity compared with other OCT systems i.e. timedomain OCT (TD-OCT), and therefore is widely investigated [3,4]. However, the relative shallow penetration depth, typically $1 \sim 3 \mathrm{~mm}[4]$, significantly limits the possible application scope of SS-OCT.

Experimental studies have demonstrated that long-wavelength band (i.e. longer than $1500 \mathrm{~nm}$ ) can increase OCT imaging penetration depth [4]. However, due to the significant amount of chromatic dispersion introduced by the additional km-long single-mode fiber (SMF) at that long-wavelength band inside the cavity, resolution, which is another key parameter of OCT, is greatly compromised. The material dispersion of the fiber places tight constraints on the possible achievable maximum sweeping bandwidth of FDML swept laser, and therefore would significantly degrade the achievable axial resolution of OCT due to the inverse relationship between the resolution and the sweeping bandwidth [5]. Several studies have been conducted to gain insight of influence of dispersion on the FDML swept laser performances [6,7]. However, practical approaches to tackle the dispersion issue in the FDML swept laser cavity at long-wavelength band has not been demonstrated yet.

In this paper, we developed a FDML swept laser configuration at long-wavelength band that allows for broad sweeping bandwidth by engineering the dispersion in the cavity. The full width at half maximum (FWHM) of the FDML swept laser sweeping bandwidth can be broadened by 33\% compared with the case without dispersion control. Therefore, the obtained OCT axial resolution determined by the spectrum is improved by a factor of $30 \%$ experimentally.

\section{Principles and Design}

In FDML swept laser, a spool of SMF delay line is employed to shift the optical round-trip frequency down to several tens of $\mathrm{kHz}$ to synchronize with the sweeping frequency of the tunable filter. The commonly used SMF in the FDML swept laser cavity, however, exhibits strong chromatic dispersion especially at the long-wavelength band and will therefore influence the achievable lasing wavelength range. This can be illustrated by the following explanations. Assuming the driving period of the tunable filter $\tau_{s w}$ perfectly synchronizes with the round-trip time $\tau_{r t}\left(\lambda_{c}\right)$ of one wavelength, which is always denoted by a central wavelength $\lambda_{c}$. Let the transmission band of the tunable filter at time $t_{1}$ to be centered at $\lambda_{1}$ as shown in Fig. 1 (a). After one round-trip time $\tau_{r t}\left(\lambda_{1}\right)$, wavelength $\lambda_{1}$ will rearrive at the tunable filter. However, due to the time mismatch of $\tau_{s w}$ and $\tau_{r t}\left(\lambda_{1}\right)$ caused by chromatic dispersion, the wavelength window $\lambda_{1}$ will not overlap exactly with the transmission band of the tunable filter, and therefore will introduce extra losses. The time mismatch after one round-trip for wavelength $\lambda_{1}$ thus can be expressed as the integral $\tau\left(\lambda_{1}\right)=\ell \int_{\lambda_{1}}^{\lambda_{c}} D\left(\lambda^{\prime}\right) d \lambda^{\prime}$ [7], where $D\left(\lambda^{\prime}\right)$ denotes the specific dispersion at the wavelength $\lambda^{\prime}$, and $\ell$ is the total fiber length of the whole cavity. This time mismatch effect will aggregate during the entire photon cavity lifetime until the cavity reaches a steady state. Extra losses would be introduced by this time mismatch effect. As lasing only occurs at the wavelength where the gain provided by the amplifier exceeds the total loss of the whole cavity, those extra losses will impact the achievable lasing wavelength range. 

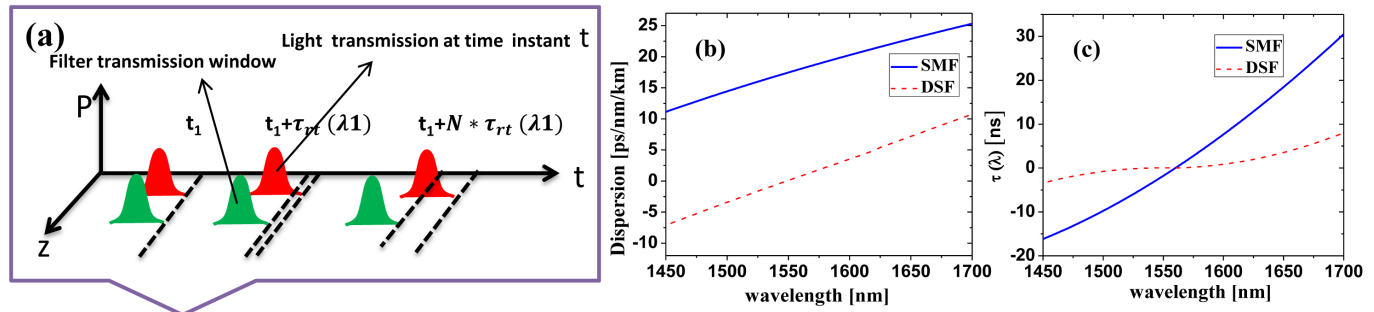

Fig. 1: (color online) (a) Illustration of mismatch effect in FDML cavity caused by dispersion. Filter transmission window (green) and light transmission at time instant t (red). (b) Dispersion of the DSF (red dashed line) and SMF (blue solid line). (c) Relative one round-trip time mismatch of DSF (red dashed line) and SMF (blue solid line) in the cavity.

In our configuration, the total dispersion of the FDML swept laser cavity is engineered by utilizing dispersionshifted fiber (DSF). Dispersion within the whole sweeping range is minimized. Therefore, the bandwidth-limiting effects [7] caused by dispersion in the standard SMF in FDML swept laser cavity are eliminated.

\section{Experimental Setup}

Fig. 2 (a) shows a schematic diagram of the setup for the FDML swept laser. The ring cavity consisted of a semiconductor optical amplifier (SOA by InPhenix) as a broadband laser gain medium. A fiber coupled piezoelectric transducer (PZT) actuated fiber Fabry-Perot tunable filter (FFP-TF by Micron Optics) was incorporated as a narrow band filter for active wavelength selection. The FFP-TF, which has a free spectral range of $200 \mathrm{~nm}$ at $1550 \mathrm{~nm}$ and a finess of 1000 , was tuned periodically at the cavity round-trip time. Two isolators were inserted to ensure unidirectional lasing. The output coupler provided 50\% output. The fiber delay line was a spool of 10-km DSF, which corresponded to a sweeping frequency of $21 \mathrm{kHz}$.
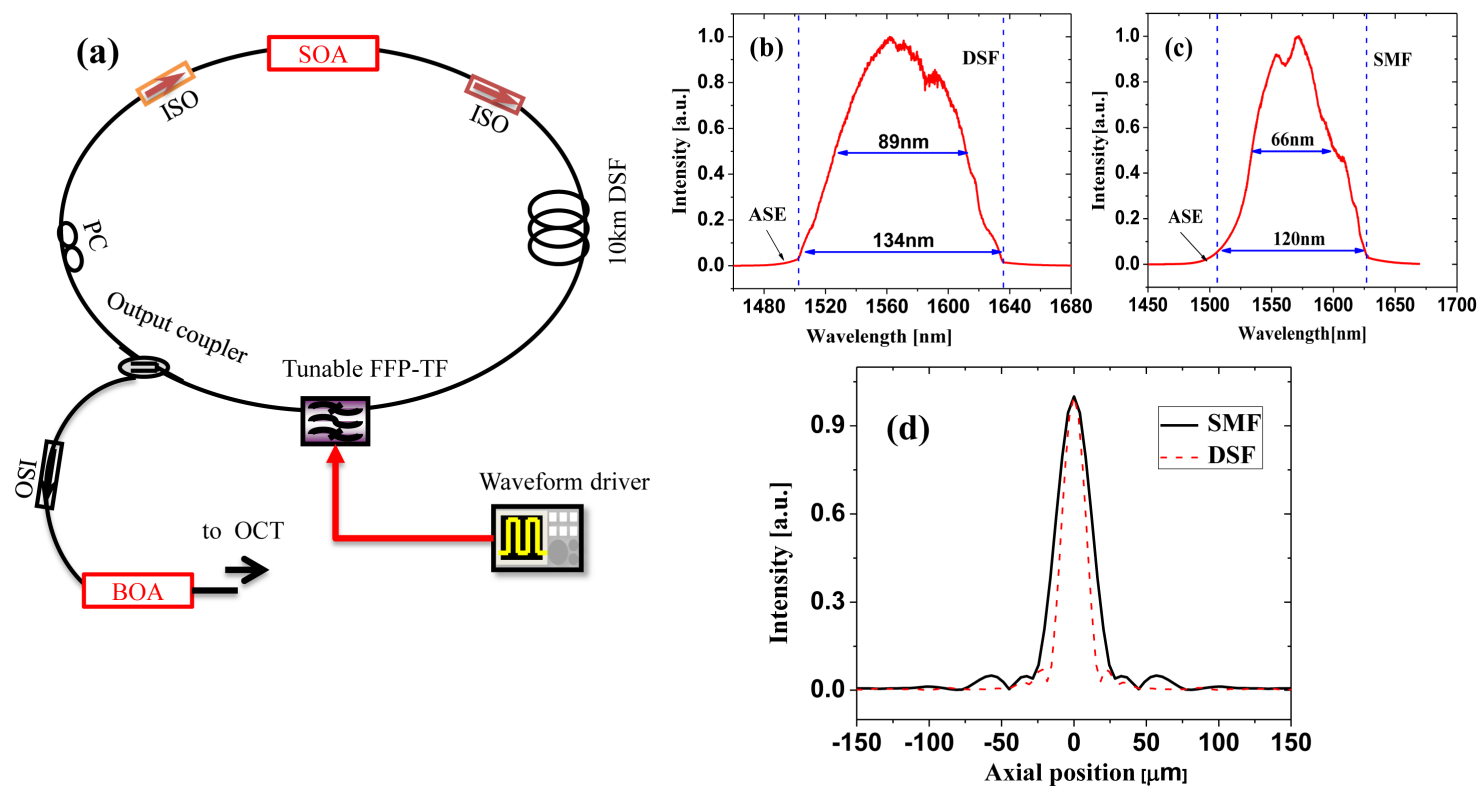

Fig. 2: (color online) (a) Schematic diagram of the FDML swept laser ring cavity. SOA: semiconductor optical amplifier. FFP-TF: fiber FabryPerot tunable filter. DSF: dispersion-shifted fiber. ISO: isolator. PC: polarization controller. BOA: booster optical amplifier. (b) Output spectrum employing dispersion-shifted fiber. (c) Output spectrum employing single-mode fiber. (d) Point spread functions of FDML swept laser utilizing DSF (black solid line) and SMF (red dashed line).

In this experiment, we investigated the influence of dispersion on the FDML operation by employing DSF. The DSF utilized in the setup has a zero-dispersion wavelength of $1549 \mathrm{~nm}$ and a dispersion slope of $0.069 \mathrm{ps} / \mathrm{nm}^{2} / \mathrm{km}$. We further investigated the quality of our scheme by replacing the DSF with commonly used SMF of same fiber length with zero-dispersion wavelength at $1310 \mathrm{~nm}$, and a dispersion slope of approximately $0.092 \mathrm{ps} / \mathrm{nm}^{2} / \mathrm{km}$ as shown in Fig. 1(b). Note that in both Fig. 1 (b) and (c), the sweeping frequency was assumed to be synchronized with the central wavelength at $1560 \mathrm{~nm}$, which was the ASE peak wavelength of the SOA. The longest one round-trip time mismatch in the range of interest (1501-1640 nm) was calculated to be $\sim 2 \mathrm{~ns}$ by utilizing DSF, while as high as $\sim 16 \mathrm{~ns}$ when 10-km DSF was replaced with 10-km SMF. Compared with SMF, the round-trip time mismatch introduced by DSF 
was significantly reduced and could be neglected within the whole gain range of SOA as shown in Fig. 1 (c).

\section{Results and Discussions}

The average output power of the FDML swept laser was measured to be $250 \mu \mathrm{W}$ when the sweeping frequency was $21 \mathrm{kHz}$. Booster optical amplifier was added after FDML as a post-amplifier to obtain improved output power. Fig. 2 (b) and (c) show the output spectrum of the FDML swept laser. When dispersion in the cavity was managed, the total sweeping range was spanned from $1503 \mathrm{~nm}$ to $1637 \mathrm{~nm}$ with a FWHM bandwidth of $89 \mathrm{~nm}$. While the spectrum covered from $1507 \mathrm{~nm}$ to $1627 \mathrm{~nm}$ with a FWHM bandwidth of only $66 \mathrm{~nm}$ if the DSF was replaced with a spool of SMF of same length. The main distinction was attributed to the dispersion difference of the cavity in the two cases. As extra dispersion-introduced loss was determined by the dispersion, the time mismatch caused by chromatic dispersion in SMF would significantly impact the effective sweeping bandwidth. It was also interesting to note the dissimilarity of the spectrum of two cases, which showed a Gaussian shaped spectrum when DSF was utilized to engineer the dispersion in the cavity. Compared with the case employing SMF, utilizing DSF enabled a much wider sweeping bandwidth and thus potentially increased the available axial resolution of the OCT system using this FDML swept laser. Further improvement of FWHM bandwidth was limited by the gain bandwidth provided by the SOA. It could be predicted that a gain medium with wider gain bandwidth could be able to provide wider effective sweeping range.

To further verify the potential axial resolution improvement of OCT system using our proposed scheme, we measured the coherence length, which was determined by backreflected signal amplitude from a single mirror reflection, of the FDML swept laser. Fig. 2 (d) shows the point spread function (PSF) obtained by Fourier transforming the interference fringe signals of the FDML swept laser. The coherence length, which corresponds to axial resolution in OCT system, is defined as the 3-dB intensity width of the PSF. In theory, the 89-nm bandwidth and 66-nm bandwidth centered at $1560 \mathrm{~nm}$ corresponded to a coherence length of $\sim 12 \mu \mathrm{m}$ and $\sim 17 \mu \mathrm{m}$ respectively in air, and therefore corresponded to a decrease in coherence length, or an improvement of axial resolution of $26 \%$. The measured values, as suggested in Fig. 2 (d), are $19 \mu \mathrm{m}$ and $27 \mu \mathrm{m}$, respectively. This is in reasonably good agreement with the theoretical prediction. The slight degradation of the measured coherence length compared with the theoretical calculation may inherit from the signal jitter and frequency nonlinearity [3] as well as the process of the numerical spectral shaping [6]. The obtained axial resolution employing DSF was enhanced by a factor of $30 \%$ which was slightly higher than theoretical calculations compared with the case utilizing SMF. This was partly due to the distorted output spectrum from the Gaussian shape when SMF was utilized, which was shown in Fig. 2 (c). Whereas perfect Gaussian shaped spectrum was assumed when theoretical calculations were performed. It could also be observed that in the case of DSF there was less side lobes owing to the Gaussian shaped spectrum.

\section{Conclusion}

We have proposed and demonstrated a novel dispersion-managed swept laser based on FDML structure. By using a dispersion-managed delay line in the cavity, the round-trip time mismatch could be minimized. The effective sweeping bandwidth was enhanced by a factor of 33\% and the obtained OCT axial resolution was improved by $30 \%$. By pursing different optical amplifiers, the sweeping range and the axial resolution is expected to be further improved. This scheme has the potential to be a cost-effective method to generate ultra-wide bandwidth FDML swept laser for ultra high resolution OCT imaging at long-wavelength band.

\section{Acknowledgement}

The work described in this paper was partially supported by grants from Research Grants Council of the Hong Kong Special Administrative Region, China ( project No. HKU7179/08E and HKU 7183/09E).

\section{References}

[1] R. Huber, M. Wojtkowski, and J. Fujimoto, "Fourier Domain Mode Locking (FDML): A new laser operating regime and applications for optical coherence tomography," Optics Express 14, 3225-3237 (2006).

[2] B. R. Biedermann, W. Wieser, C. M. Eigenwillig, T. Klein, and R. Huber, "Dispersion, coherence and noise of Fourier domain mode locked lasers," Optics Express 17, 9947-9961 (2009).

[3] R. Huber, K. Taira, M. Wojtkowski, T. H. Ko, J. G. Fujimoto, and K. Hsu, "High speed frequency swept light source for Fourier domain OCT at $20 \mathrm{kHz}$ A-scan rate," Photonics West-Bios (2005).

[4] U. Sharma, E. W. Chang, and S. H. Yun, "Long-wavelength optical coherence tomography at $1.7 \mathrm{~m}$ for enhanced imaging depth," Optics Express 16, 19712-19723 (2008).

[5] J. M. Schmitt, "Optical coherence tomography (OCT): a review," Selected Topics in Quantum Electronics, IEEE Journal of 5, 1205-1215 (1999).

[6] R. Huber, D. Adler, V. Srinivasan, and J. Fujimoto, "Fourier domain mode locking at $1050 \mathrm{~nm}$ for ultra-high-speed optical coherence tomography of the human retina at 236,000 axial scans per second," Optics letters 32, 2049-2051 (2007).

[7] S. Marschall, T. Klein, W. Wieser, B. R. Biedermann, K. Hsu, K. P. Hansen, B. Sumpf, K. H. Hasler, G. Erbert, and O. B. Jensen, "Fourier domain mode-locked swept source at $1050 \mathrm{~nm}$ based on a tapered amplifier," Virtual Journal for Biomedical Optics 5 (2010). 